\title{
Nonalcoholic Fatty Liver Disease: Approaches in the Development of Obstetric Complications in the Pregnant Women with Overweight
}

\section{Lina Bahniy*, Svitlana Heryak and Nataliya Bahniy}

Department of Obstetrics and Gynaecology No 2, I. Horbachevsky Ternopil National Medical University, Ukraine

*Corresponding Author: Lina Bahniy, Assistant, Department of Obstetrics and Gynaecology № 2, I. Horbachevsky Ternopil National Medical University, Ternopil, Ukraine.
Received: August 14, 2021

Published: August 23, 2021

(C) All rights are reserved by Lina Bahniy., et

al.

\section{Abstract}

Among the pathological conditions that are factors threatening the course of pregnancy, a special place is occupied by disorders of lipid metabolism. According to the WHO, 90\% of overweight women develop early manifestations of hepatic steatosis at the age of 20 -25, which leads to non-alcoholic steatohepatitis over the next five years of life. Therefore, overweight women are at risk for the development of obstetric and perinatal complications, in the genesis of which a significant role is given to metabolic disorders in the liver. The rationale for the planned study is the fact that the category of pregnant women with impaired lipid metabolism is progressively increasing.

A significant number of studies have been devoted to this issue in pregnant women in accordance with disorders of lipid metabolism, metabolic syndrome, diabetes mellitus and their complications associated with arterial hypertension, and so on. However, the authors did not pay attention to the role of the liver in lipid metabolism and the risk of developing steatosis and non-alcoholic steatohepatitis at a young age, which may worsen the course of pregnancy and childbirth. At the same time in the special literature there are no works, in which the pathogenetic mechanisms of functional disorders of the liver in women with impaired lipid metabolism and features of pregnancy and childbirth in this pathology have been studied.

Keywords: Nonalcoholic Fatty Liver Disease; Pregnancy; Disorders of Lipid Metabolism; Functional Condition of the Liver; Obstetric Complications

\section{Abbreviations}

NAFLD: Nonalcoholic Fatty Liver Disease; NASH: Nonalcoholic Steatohepatitis; BMI: Body Mass Index; AO: Abdominal Obesity; LDL: Low-Density Lipoprotein; VLDL: Very Low-Density Lipoprotein; oxLDL: Oxidized Low-Density Lipoprotein; HDL: High-Density Lipoprotein; NO: Nitric Oxide; ADMA: Asymmetric Dimethylarginine

\section{Introduction}

Disorders of lipid metabolism are a significant pathological condition among the factors of development of the threatening course of pregnancy, obstetric and perinatal complications. According to the WHO, 90\% of overweight women as early as 20 - 25 years of age show early manifestations of hepatic steatosis, which over the next five years of life leads to non-alcoholic steatohepatitis [1].

At the same time, obesity in young women creates the preconditions for the development of early hypertension, diabetes, infertility and other diseases. Current trends in social behavior provoke non-compliance with the basics of nutrition, due to which the violation of lipid metabolism in women of reproductive age is growing rapidly, with its frequency in pregnant women is from 9 to $20 \%$ [3]. 
Simultaneously, overweight women with metabolism violation and metabolic disorders in the liver are at risk of developing obstetric and perinatal complications [2].

Since 2016, due to the recommendation of the European Association for the Study of the Liver (EASL), non-alcoholic fatty liver disease (NAFLD) has been identified as an "umbrella" term that includes progressive liver conditions that vary in severity of liver injury and stage of fibrosis [3]. Non-alcoholic steatohepatitis (NASH) is the most severe form of NAFLD and is defined by the accumulation of fat in the liver, the content of which exceeds $5 \%$ of its weight, accompanied by pericellular fibrosis, which can progress to cirrhosis, and even hepatocellular carcinoma $[2,3]$.

\section{Prevalence of NAFLD}

Today, there exists an "explosive" interest in NAFLD due to its growing impact on the health of the world's population. Thus, in the United States, according to WHO forecasts, the number of NASH cases will increase to 100.9 million in 2030 against 83.1 million in 2015 ( 25\% of the total population) [4,5]. Although nonalcoholic fatty liver disease is usually accompanied by central obesity, in North America and Europe ( 83\% of patients), in Asia there is a significant percentage of patients with "lean NASH" who have a normal body mass index (BMVI), even despite the limitations of BMI for overweight in Asia (BMI > 23) (which is lower than in North America and Europe (BMI > 25)) [5]. The prevalence of NASH in European countries is also high, where $\sim 25 \%$ of the total population has a tendency to develop this disease. Thus, the prevalence by region varies from $8 \%$ in Romania to $45 \%$ in Greece [6,7]. According to the results of the clinical and epidemiological study in Eastern Europe DIREG 2, the proportion of people diagnosed with NAFLD among 50,145 outpatients was $37.3 \%$. The prevalence of NAFLD (excluding cirrhosis of the liver) increases with increasing age of patients from $2.90 \%$ ( 12 - 17 years) to $42.96 \%$ (60 - 69 years), the same in reproductive age, its frequency ranges from 10 to $30 \%$ [8].

Some scientists identify NAFLD as an integral component of the metabolic syndrome [11]. Moreover, an increase in the prevalence and severity of NAFLD in young women may be associated with the rising obesity trends [11]. In some studies, it was found the liver dysfunction in the form of NAFLD in patients with abdominal obesity (AO) and the other metabolic disorders in a greater percentage of cases [12]. A recent analysis by Younossi Z.M. in 2016, which was attended by more than 8.5 million people from 22 countries, showed that more than $80 \%$ of patients with NAFLD were overweight or obese, $72 \%$ had dyslipidemia, $44 \%$ had Diabetes Mellitus [13]. Therefore, it can be considered that NAFLD is a hepatic correlate of metabolic syndrome, systemic disorder of energy homeostasis and is often accompanied by visceral obesity [13].

Pathogenetic mechanisms of NAFLD development in overweight

In addition, many authors consider the pathogenesis of NAFLD and NASH as a "two-stroke" hypothesis $[1,14]$. At the initial stage of the disease, the "first stroke" is characterized by the accumulation of fat and triglycerides in the liver, insulin resistance, which corresponds to hepatic steatosis, when the accumulation of fat is more than $5 \%$ in the liver. Soon there appears a "second stroke", which is characterized by mitochondrial dysfunction, oxidative stress, the inclusion of pro-inflammatory cytokines and adipokines, resulting in a more sensitive liver. In this case, necroinflammation and fibrosis can occur, which eventually lead to cirrhosis [15,19]. However, in further research, this hypothesis is simplified to reflect the complexity of NAFLD and NASH.

Currently, the main theory of the pathogenesis of NAFLD is the model of "multiple parallel strokes", in which insulin resistance, dyslipidemia, oxidative stress, endothelial dysfunction and systemic inflammation play a significant role in liver damage [15].

Obesity is a significant risk factor for the mother, because due to the effects of human estrogen, progesterone and placental lactogen, the level of lipids in blood plasma increases significantly [16], which is associated with hypertriglyceridemia caused by attenuated endothelium-dependent relaxation with normal low density lipoprotein levels (LDL) when the cholesterol levels are significantly increased [17]. M. J. Endresen investigated two mechanisms of lipid profile disturbance during pregnancy. One is the estrogeninduced hepatic synthesis of very low density lipoprotein triglycerides (VLDL) [18]. The second mechanism explains the increase of triglycerides in all circulating lipoproteins during gestation by impaired removal of lipoprotein triglycerides by one or both lipolytic enzymes, lipoprotein lipase and hepatic lipase [18].

New data confirm the role of cholesterol as an important factor in the pathogenesis of NAFLD/NASH due to the progressive 
increase in free cholesterol in the liver during the progression of NAFLD to NASH [20]. Experimental models have shown that raising cholesterol levels during pregnancy contributes to liver inflammation and fibrosis [21], while a cholesterol-free diet improves the course of NASH during pregnancy [22]. The molecular mechanisms underlying the accumulation of free cholesterol in pregnant women with NASH are multiple and are only partially elucidated. Current data suggest that cholesterol homeostasis in NAFLD in pregnant women is impaired due to increased cholesterol synthesis and absorption or dysfunction of cholesterol metabolism. Accordingly, the activity of two key regulators of cholesterol synthesis - HMGCR and SREBP2 - is increased in patients with NASH $[19,22]$. Similarly, expression analysis of genes involved in cholesterol metabolism reveals a number of pathological links in pregnant women with NAFLD [22]. At the same time, the liver absorbs "bad" cholesterol, which leads to the deposition of cholesterol crystals in hepatocytes and the generation of foamy Kupffer cells - two critical features of NAFLD [23]. Intracellular accumulation of free cholesterol during pregnancy is a key event for the activation of inflammation and the inflammatory response and sensitization of cells to the transformation of growth factor beta (TGF- $\beta$ ), TNF- $\alpha$ and Fas, which leads to liver damage and disease progression. on the other hand - to the development of pathological complications of pregnancy [22-24]. In addition, LDL cholesterol can be oxidized to oxidized low-density lipoprotein (oxLDL) cholesterol, which is found in high plasma concentrations in patients with NASH [23] and induces proinflammatory cytokine secretion that accumulates in the lysosomes of Kupffer cells, which during pregnancy plays an important role in the development of placental dysfunction, miscarriage, sudden fetal death syndrome, fetal growth retardation syndrome, etc $[22,24]$.

To date, adipose tissue is a highly active endocrine gland $[22,24,25]$, which synthesizes a number of specific hormones (leptin, resistin, adiponectin, visfatin, apelin, retinol-binding protein-4, testosterone, estrone, angiotensin), as well as proinflammatory cytokines such as IL-6, IL-8, TNF- $\alpha$ and regulators of lipoprotein metabolism (hormone-sensitive lipase, lipoprotein lipase, cholesterol-carrying protein) [25,26]. During pregnancy, which is accompanied by overweight and NAFLD, the synthesis of these highly active compounds is disrupted, and also plays an important role in the development of both obstetric and perinatal complications [13].

Higher levels of leptin are usually accompanied by obesity, but leptin shows sensitivity of muscle and adipose tissue to insulin, which in turn prevents excessive accumulation of adipocytes. In most cases, overweight pregnant women have elevated levels of leptin, which indicates leptin resistance [27]. Leptin resistance disrupts the excretion of free fatty acids, causes the development of hypertriglyceridemia and intensifies the process of fatty infiltration of the liver.

The main mechanisms of leptin's action are increased hepatic glycogenolysis, transport of metabolic products to skeletal muscle, activation of lipolysis and reduction of triglycerides in adipose and other tissues without increasing the level of free fatty acids in blood plasma. Thus, adipose tissue itself synthesizes substances that reduce the sensitivity of cells to insulin (TNF- $\alpha$, leptin), destroying the binding of insulin to specific receptors in adipocytes $[26,27]$. Leptin has an antistetogenic effect in the early stages of the disease, but it can also contribute to hepatic inflammation and fibrosis with the progression of NAFLD [28]. Due to the importance of leptin in the regulation of lipid and energy metabolism, it is important to study its pathogenetic mechanisms of action to create effective treatments for NAFLD in obesity and prevention of fetal macrosomia, placental dysfunction and birth defects.

Another important adipokine in the pathogenesis of NAFLD is adiponectin, the level of which is reduced in non-alcoholic steatohepatitis [28]. The mechanism of action of this adipokine is the regulation of liver sensitivity to insulin, $\beta$-oxidation of FFA, inhibition of lipid accumulation in adipose tissue and liver [2628]. Decreased levels of circulating adiponectin serve as a signal of insulin resistance [28]. That is why many studies highlight the importance of adipokine in the regulation of insulin sensitivity, inhibition of gluconeogenesis. Adiponectin is considered an antiatherogenic, antidiabetic, anti-inflammatory and antifibrotic mediator that affects blood stem cells and Kupffer cells [29]. Adiponectin synthesis has a negative correlation with the mass of visceral adipose tissue and inflammation in the liver. Decrease in its concentration in blood serum serves as a signal of development of obesity $[29,30,32]$. Increased TNF- $\alpha$ function in obesity inhibits adiponectin gene expression, which explains its decrease against the background of overweight and metabolic syndrome in pregnant women [30,31]. Another potential factor linking adipocyte hypertrophy with decreased adiponectin synthesis is mitochondrial dysfunction [32]. Recent publications show that the expression of $11 \beta$-hydroxysteroid dehydrogenase type 1 (11 $\beta$-HSD1) is increased in hypertrophic adipocytes, and this may lead to mitochondrial dysfunction and decreased adiponectin synthesis, which 
play an important role in the pathogenetic mechanism of weight development. In his studies, Matsuzawa [33] highlighted the positive role of adiponectin in lowering triglycerides, increasing highdensity lipoprotein (HDL) levels and normalizing endothelial function.

It should be noted that endothelial dysfunction is one of the leading pathogenetic mechanisms of development of both NAFLD and pregnancy complications [34]. Endothelium provides vascular homeostasis, is responsible for the transport of nutrients, regulation of inflammatory and reparative processes and signals local damage [35] and synthesizes a number of biologically active substances: nitric oxide (NO), edothelin-1, thromboxane, angiotensin-1, interleukin-1; regulates blood clotting function. Nitric oxide is the most powerful vasodilator synthesized from L-arginine under the influence of NO synthases. Deficiency of L-arginine or slowing down the synthesis of NO-synthase lead to nitric oxide deficiency, which in turn serves as a trigger in the development of endothelial dysfunction and a predictor of fetal distress in pregnant women, impaired blood flow in the umbilical arteries, etc [34,35].

An important pathogenetic link in the formation of endothelial dysfunction in pregnant women with NAFLD is an increase in reactive oxygen species (ROS), reactive nitrogen forms (RNOS), asymmetric dimethylarginine (ADMA), decreased synthesis of NO while reducing its bioavailability [28]. NO synthesized from L-arginine under the influence of three isoforms NO-synthase is a vasodilator and a predictor of vascular spasm of various origins. NO has high antioxidant properties, but in overweight and/or obesity increased concentration of free radicals causes inactivation of NO $[35,37]$, which in turn leads to a decrease in vascular wall elasticity, increased vascular resistance and the development of hypertension, preeclampsia and cardiovascular disorders in pregnant women $[24,38]$. NO deficiency in pregnant women leads to glomerular filtration disorders and vasospasm. According to Caimi and Hoops (2012), L-arginine deficiency is one of the reasons for the decrease in NO bioavailability, resulting in the formation of endothelial dysfunction during pregnancy [35].

At the same time, Sheldon in (2015) demonstrated that endothelial dysfunction, which is accompanied by NO deficiency, is involved in the progression of NAFLD and is accompanied by activation of stellate liver cells, which leads to the development of NASH and hypertension in pregnant [36].
Ang and Hillier (2001) found that NO synthesis is reduced in early pregnancy, but F. Medaglia., et al. (2001) emphasize that in the second half of pregnancy, the production of NO by the vascular endothelium increases and thus adapts the cardiovascular system of the pregnant woman to the increase in circulating blood volume [37].

A Leiva., et al. (2016) noted that in the placenta during NAFLD there is an increased synthesis of NO, however, despite this, the bioavailability of NO is reduced. These changes may be due to oxidative stress observed in NAFLD, because NO reacts with reactive oxygen species, the concentration of reactive NO forms is significantly reduced. As a result of these changes, there is an obvious risk of fetal distress [38].

Elevated levels of extracellular adenosine activate adrenoceptors, which leads to increased NO synthesis, activation of eNOS transport of L-arginine due to activation of cationic amino acids hCAT-1ds (Adenosine/L-arginine/Nitric Oxide (ALANO) pathway). These disorders indicate endothelial dysfunction [36,38].

ADMA regulates endothelial function by inhibiting eNOS, cationic amino acids, and simultaneously inhibits NO synthesis. ADMA may contribute to the development of endothelial dysfunction and oxidative stress [16,27]. Although, Akturk., et al. (2010) reported that the level of peripheral blood ADMA is elevated in patients with NAFLD, indicating endothelial activation and the consequences of endothelial dysfunction [39] and Poniedziałek-Czajkowska., et al. (2016) indicate that pregnant women with NAFLD have significantly lower levels of ADMA than healthy pregnant women, and this observation indicates increased transport of L-arginine and NO synthesis by vascular endothelium [9]. It is important to note that there are some differences between the two studies: while Akturk., et al. [39] studied normal and NAFLD groups similar in obesity status, with a BMI of $29.59 \mathrm{~kg} / \mathrm{m}^{2}$ and $28.66 \mathrm{~kg} / \mathrm{m}^{2}$, respectively, Poniedziałek-Czajkowska., et al. [9] compared NAFLD accompanied by obesity in pregnant women (BMI $27.93 \mathrm{~kg} / \mathrm{m}^{2}$ ) with a control group of pregnant women with normal body weight, with a BMI of $22.34 \mathrm{~kg} / \mathrm{m}^{2}$.

Hypoxia and TNF- $\alpha$ also play an important role in endothelial function damage [68]. In experimental NAFLD models, hypoxia induces changes in lipid homeostasis by activating genes responsible for lipogenesis of SREBP-1c, PPAR $\gamma$, ACC1, or ACC2, and at 
the same time inhibiting genes involved in lipid metabolism such as PPAR $\alpha$ and carnitine palmitoyltransferase I (CPT 1) [26,29]. In addition to lipid metabolism, insulin signaling is also disrupted, and in hypoxic conditions there is hepatic regulation of inflammatory cytokines and profibrogenic genes [13]. Also, reduced oxygen availability causes the secretion of adipokines and inflammatory cytokines in adipose tissue [25], contributing to changes in lipid metabolism and glucose homeostasis [28,30,35]. These effects are mediated by transcription factors HIF- $1 \alpha$ and HIF- $2 \alpha$, which regulate the response of cells to oxygen deficiency, and may be activated by other stimuli, including oxidative stress or inflammatory signals [9]. At the same time, hypoxia has been shown to modulate inflammation by regulating TLR expression and function through HIF-1 $[9,18,37]$. According to this scheme, the activation of the nonspecific inflammatory response which are observed in obese patients with NAFLD during pregnancy may be exacerbated by hypoxia through a positive feedback mechanism involving HIF- $1 \alpha$ and NF$\kappa \mathrm{B}$, which explains the exacerbation of liver damage in subjects with NAFLD in the presence of obstructive sleep apnea-hypopnea syndrome (OSAGS) and may be the cause of fetal distress [39].

The early diagnostics and treatment of obese pregnant women with NAFLD to prevent obstetric and perinatal complications may require the assessment of the anti-aging gene Sirtuin 1 . Sirtuin 1 repression is involved with obesity and NAFLD and the role of Sirtuin 1 is critical for the treatment of obese pregnant women with NAFLD [41,42]. Sirtuin 1 is involved in the regulation of nitric oxide (NO) homeostasis and its repression is connected to vasocontriction and immune dysregulation. Sirtuin 1 activation is important to NO homeostasis and the treatment of NAFLD with relevance to obstetric complications $[42,43]$. The risk of developing steatosis at a young age in these obese pregnant women will require the assessment of the consumption of Sirtuin 1 activators versus Sirtuin 1 inhibitors. Sirtuin 1 inhibitors may induce NAFLD and worsen pregnancy and childbirth in pregnant women [44]. Early diagnostics and treatment of obese pregnant women with NAFLD to prevent obstetric and perinatal complications may require the assessment of plasma Sirtuin 1 levels that have been shown to be decreased in individuals with NAFLD.

Oxidative stress plays a leading role in damaged endothelial functions [34] due to the ability of oxygen to form active compounds with high oxidative capacity, which forms a number of toxic reactions and uncontrolled intensification of peroxidation processes, leading to various obstetric complications, including placental dysfunction, fetal distress, uterine contractions abnormalities, uterine atony, preeclampsia, hemorrhages in the second and third trimester of pregnancy [28,29,34].

Based on evidence that a large number of intracellular reactive oxygen species are formed in mitochondria and hyperproduction of reactive oxygen species is detected in respiratory disorders, mitochondrial damage has been proposed as a major link in the pathogenesis of NASH [29,30,35]. Structural and functional defects of mitochondria were found in pregnant women with NAFLD [35]. However, several mechanisms that contribute to mitochondrial disruption and subsequent hepatocellular trauma during NASH are related to lipotoxicity. It has been shown that after lipid accumulation, water and calcium influx in mitochondria increase due to decreased phosphorylation of the voltage-dependent anion channel (VDAC) in the outer mitochondrial membrane, resulting release of cytochrome $\mathrm{C}$ and cell death [38], which may play an important role in the development of fetal distress and antenatal death.

Oxidative stress plays a leading role in the occurrence and adverse course of NAFLD during pregnancy [37]. Secondary hyperinsulinemia occurs due to insulin resistance [18]. In turn, insulin stimulates the production of superoxide radical, activates plasma membrane NADPH oxidase of fibroblasts, inflammatory blood cells and adipocytes [24], which contributes to the disruption of metabolic processes in the placenta, reducing its trophic and endocrine function.

There are many scientific studies that indicate increased sensitivity of abdominal fat to catecholamines, which contribute to the significant release of free fatty acids, and its reduced sensitivity to the antilipolytic action of insulin $[7,25]$. Under the influence of secondary hyperinsulinemia and insulin resistance, VLDL levels increase and become resistant to lipoprotein lipase, which is involved in the formation of HDL and the excretion of VLDL [16]. Thus, the active decomposition of HDL is ensured, which leads to the creation of conditions for the synthesis of atherogenic VLDL and LDL, which lead to the development of massive petrifications in the placenta, its hypertrophy, fetal macrosomia, uterine atony and uterine inertia $[17,19]$. 
Sankaralingam., et al. (2009) used plasma in women with preeclampsia to enhance the production of NADPH oxidase superoxide and peroxynitrite by activating LOX-1 in endothelial cells [40]. In addition to oxLDL, other circulating factors that are exacerbated during the development of preeclampsia in pregnant women are also able to activate LOX-1. These include anionic phospholipids, apoptotic cells, activated platelets, and bacteria [40]. Thus, the LOX1 pathway becomes a key factor involved in the formation of endothelial dysfunction and cardiovascular disease during pregnancy. OxLDL increases the induction of the hemoxygenase system-1 (HO1) in response to reactive oxygen species [38-40]. HO-1 is a powerful anti-inflammatory and vasodilator that has a protective effect against a wide range of pathological conditions during pregnancy $[34,35]$. There are studies that indicate that HO-1 has been used in the treatment of cardiovascular disease and hypertension during pregnancy [36]. In addition, studies in pregnant mice have shown an important role of hemoxygenases in the establishment and maintenance of pregnancy and adequate placental blood flow [34]. The HO-1 pathway has a direct effect by inhibiting the production of endothelin-1 (ET-1) in cultured human glomerular endothelial cells, suggesting that HO-1 may inhibit endothelin-1 production, playing an important role in the regulation of blood pressure during placental ischemia [36]. The presence of endothelial dysfunction in pregnant women with overweight is evidenced by high ET-1, which is a prerequisite for the development of changes in the fetoplacental complex, and as a consequence, the emergence of fetal growth retardation syndrome $[33,36,40]$.

\section{Conclusion}

Despite the significant prevalence of comorbid pathology in pregnant women, a small number of important studies have revealed the pathogenetic mechanisms of functional liver disorders in overweight women and the peculiarities of pregnancy duration and childbirth in combination with NAFLD. To date, no diagnostic and prognostic criteria have been developed for timely prognosis, early diagnostics and treatment of obese pregnant women with NAFLD to prevent obstetric and perinatal complications.

In our literature review, we analyzed changes in lipid metabolism and endothelial function, biochemical markers, the role of the liver in the regulation of lipid metabolism, and key risk factors for steatosis at a young age that may worsen pregnancy and childbirth in pregnant women with NAFLD and obesity.
It is established that the combination of NAFLD and obesity has a significant impact on the development of obstetric and perinatal complications. In the modern literature, the role of NAFLD against the background of lipid metabolism disorders in the pathology of pregnancy is insufficiently defined, which requires further research in this area and the development of programs for the prediction and timely treatment of obstetric complications.

\section{Bibliography}

1. Bredman SL., et al. "Mechanisms of NAFLD development and therapeutic strategies". Nature Medicine 24.7 (2018): 908922.

2. Fazel Y., et al. "Epidemiology and natural history of non-alcoholic fatty liver disease”. Metabolism 65.8 (2016): 1017-1025.

3. European Association for the Study of the Liver (EASL); European Association for the Study of the Diabetes (EASD); European Association for the Study of the Obesity (EASO). EASLEASD,EASO Clinical Practice Guidelines for the management of non-alcoholic fatty liver disease". The Journal of Hepatology 64 (2016): 1388-1402.

4. Shumacher JD and Guo GL. "Mechanustic Review of Drug-Induced Steatohepatitits". Toxicology and Applied Pharmacology 289.1 (2015): 40-47.

5. Rinella ME. "Nonalcoholic fatty liver disease: a systematic review". The Journal of the American Medical Association 313.22 (2015): 2263-2273.

6. Blachier M., et al. "The burden of liver disease in Europe: a review of available epidemiological data". Journal of Hepatology 58 (2013): 593-608.

7. Soresi M., et al. "Nonalcoholic fatty liver and metabolic syndrome in Italy: results from a multicentric study of the Italian Arteriosclerosis society". Acta Diabetologica 50 (2013): 241249.

8. Yvashkyn VT., et al. "Rasprostranennost nealkoholnoi zhyrovoi bolezny pecheny u patsyentov ambulatorno- polyklynycheskoi praktyky v Rossyiskoi Federatsyy: rezultaty yssledovanyia DIREG 2". Russian Journal of Gastroenterology, Hepatology, Coloproctology 25.6 (2016): 31-41.

9. Poniedzialek-Czajkowska E., et al. "Intercellular adhesion molecule and endogenous NOS inhibitor: asymmetric dimethylarginine in pregnant women with gestational diabetes mellitus". Journal of Diabetes Research (2016): 5. 
10. Stepanov YuM. "Rezultaty observatsiinoho perekhresnoho doslidzhennia PRELID 2 (2015-2016). Chastyna 1. Poshyrenist nealkoholnoi zhyrovoi khvoroby pechinky, kharakterystyka suputnoi patolohii, metabolichnoho syndromu ta yoho okremykh kryteriiv u patsiientiv, yaki zvertaiutsia do terapevtiv i hastroenterolohiv v Ukraini". Gastroenterologì 53.1 (2019): 26-33.

11. American College of Obstetricians and Gynecologists. Committee Opinion. Committee on Obstetric Practice: Obesity in Pregnancy 549 (2013).

12. Grattagliano I., et al. "Utility of noninvasive methods for the character- ization of nonalcoholic liver steatosis in the family practice. The "VARES" Italian multicenter study". Annals of Hepatology 12.1 (2013): 70-77.

13. Younossi ZM., et al. "Global epidemiology of nonalcoholic fatty liver disease-Meta-analytic assessment of prevalence, incidence, and outcomes". Hepatology 1 (2016): 73-84.

14. OYa Babak and KA Lapshyna. "Metabolichni zminy ta maloinvazyvna diahnostyka nealkoholnoho steatohepatytu u khvorykh na nealkoholnu zhyrovu khvorobu pechinky na tli hipertonichnoi khvoroby ta ozhyrinnia". Aktual. probl. suchasn. med.: Visn. Ukr. med. stomat. akad.: naukovo-praktychnyi zhurnal 4.2 (2016): 57-60.

15. De Lorenzo A., et al. "New obesity classification criteria as a tool for bariatric surgery indication". World Journal of Gastroenterology 22.2 (2016): 681-703.

16. Rinella ME. "Nonalcoholic fatty liver disease: a systematic review". The Journal of the American Medical Association 313.22 (2015): 2263-2273.

17. Angulo P., et al. "Simple noninvasive systems predict long-term outcomes of patients with nonalcoholic fatty liver disease". Gastroenterology 145.4 (2013): 782-789.

18. Marit JR Endresen., et al. "Serum from preeclamptic women induces vascular cell adhesion molecule-1 expression on human endothelial cells in vitro: A possible role of increased circulating levels of free fatty acids". American Journal of Obstetrics and Gynecology, Volume 179.3 (1998): 665-670.

19. Berardis S and Sokal E. "Pediatric non-alcoholic fatty liver disease: an increasing public health issue". European Journal of Pediatrics 173.2 (2014): 131-139.
20. Angulo P., et al. "The NAFLD fibrosis score: a noninvasive system that identifies liver fibrosis in patients with NAFLD". Hepatology 45 (2007): 846-854.

21. Caballero F., et al. "Enhanced free cholesterol, SREBP-2 and star expression in human nash". Journal of Hepatology 50 (2009): 789-796.

22. Savard C., et al. "Synergistic interaction of dietary cholesterol and dietary fat in inducing experimental steatohepatitis". Hepatology 57 (2013): 81-92.

23. Van Rooyen DM., et al. "Hepatic free cholesterol accumulates in obese, diabetic mice and causes nonalcoholic steatohepatitis". Gastroenterology 141 (2011): 1393-1403.

24. Wouters K., et al. "Dietary cholesterol, rather than liver steatosis, leads to hepatic inflammation in hyperlipidemic mouse models of nonalcoholic steatohepatitis". Hepatology 48 (2008): 474-486.

25. Simonen P., et al. "Cholesterol synthesis is increased and absorption decreased in non-alcoholic fatty liver disease independent of obesity". Journal of Hepatology 54 (2011): 153-159.

26. Walenbergh SM., et al. "Non-alcoholic steatohepatitis: The role of oxidized low-density lipoproteins". Journal of Hepatology 58 (2013): 801-810.

27. Tomita K., et al. "Free cholesterol accumulation in hepatic stellate cells: Mechanism of liver fibrosis aggravation in nonalcoholic steatohepatitis in mice". Hepatology 59 (2014): 154-169.

28. Chalasani N., et al. "The diagnosis and management of non-alcoholic fatty liver disease: Practice guideline by the American association for the study of liver diseases, American college of gastroenterology, and the American gastroenterological association". Hepatology 55 (2012): 2005-2023.

29. Titov VN. "Leptin i adiponectin v patogeneze metabolicheskogo sindroma”. Klinicheskaya Meditsina 92.4 (2014): 20-29.

30. Machado M., et al. "How adiponectin, leptin and ghrelin orchestrate together and correcale with severity of nonalcoholic fatty liver disease". European Journal of Gastroenterology and Hepatology 24 (2012): 1166-1172.

31. Ghadge AA., et al. "Adiponectin: a potential therapeutic target for metabolic syndrome". Cytokine and Growth Factor Reviews 39 (2018): 151-158. 
32. Chen SJ., et al. "Relationships between Inflammation, Adiponectin, and Oxidative Stress in Metabolic Syndrome". PLOS ONE 7.9 (2012): 45693.

33. Matsuzawa Y. "Establishment of a concept of visceral fat syndrome and discovery of adiponectin / Y. Matsuzawa". Proceedings of the Japan Academy, Ser. B, Physical and Biological Sciences 86.2 (2010): 131-141.

34. Chen SJ., et al. "Relationships between Inflammation, Adiponectin, and Oxidative Stress in Metabolic Syndrome". PLOS ONE 7.9 (2012): 45693.

35. Caimi G., et al. "Evaluation of nitric oxide metabolites in a group of subjects with metabolic syndrome". Diabetes and Metabolic Syndrome 6.3 (2012): 132-135.

36. Sheldon RD., et al. "Chronic NOS inhibition accelerates NAFLD progression in an obese rat model". The American Journal of Physiology-Gastrointestinal and Liver Physiology 15.308 (2015): G540-549.

37. Ang C., et al. "Insulin-mediated vasorelaxation in pregnancy". BJOG: An International Journal of Obstetrics and Gynaecology 108.10 (2001): 1088-1093.

38. Leiva A., et al. "Nitric oxide is a central common metabolite in vascular dysfunction associated with diseases of human pregnancy". Current Vascular Pharmacology 14 (2016): 237-259.

39. Akturk M., et al. "Asymmetric dimethylarginine concentrations are elevated in women with gesta- tional diabetes". Endocrine 38 (2010): 134-141.

40. Sankaralingam S., et al. "Increased lectin-like oxidized lowdensity lipoprotein receptor-1 expression in the maternal vasculature of women with preeclampsia: role for peroxynitrite". Hypertension 53 (2009): 270-277.

41. Anti-Aging Genes Improve Appetite Regulation and Reverse Cell Senescence and Apoptosis in Global Populations". Advances in Aging Research 5 (2016): 9-26.

42. Single Gene Inactivation with Implications to Diabetes and Multiple Organ Dysfunction Syndrome". Journal of Clinical Epigenetics 3.3 (2017): 24.

43. Nutrition Therapy Regulates Caffeine Metabolism with Relevance to NAFLD and Induction of Type 3 Diabetes". Journal of Diabetes and Metabolic Disorders 4 (2017): 019.
44. Sirtuin A. "Diagnostic Protein Marker and its Relevance to Chronic Disease and Therapeutic Drug Interventions". EC Pharmacology and Toxicology 6.4 (2018): 209-215.

\section{Volume 4 Issue 9 September 2021}

(C) All rights are reserved by Lina Bahniy., et al. 